\title{
Joint constraints on the field-cluster mixing fraction, common envelope efficiency, and globular cluster radii from a population of binary hole mergers via deep learning
}

\author{
Kaze W. K. Wong $\odot,^{1, *}$ Katelyn Breivik, ${ }^{2}$ Kyle Kremer, ${ }^{3,4}$ and Thomas Callister ${ }^{2}$ \\ ${ }^{1}$ Department of Physics and Astronomy, Johns Hopkins University, \\ 3400 N. Charles Street, Baltimore, Maryland 21218, USA \\ ${ }^{2}$ Center for Computational Astrophysics, Flatiron Institute, New York, New York 10010, USA \\ ${ }^{3}$ TAPIR, California Institute of Technology, Pasadena, California 91125, USA \\ ${ }^{4}$ The Observatories of the Carnegie Institution for Science, Pasadena, California 91101, USA
}

(Received 25 November 2020; accepted 24 March 2021; published 23 April 2021)

\begin{abstract}
The recent release of the second Gravitational-Wave Transient Catalog (GWTC-2) has increased significantly the number of known GW events, enabling unprecedented constraints on formation models of compact binaries. One pressing question is to understand the fraction of binaries originating from different formation channels, such as isolated field formation versus dynamical formation in dense stellar clusters. In this paper, we combine the COSMIC binary population synthesis suite and the CMC code for globular cluster evolution to create a mixture model for black hole binary formation under both formation scenarios. For the first time, these code bodies are combined self-consistently, with CMC itself employing COSMIC to track stellar evolution. We then use a deep-learning enhanced hierarchical Bayesian analysis to continuously sample over and constrain the common envelope efficiency $\alpha$ assumed in COSMIC, the initial cluster virial radius $r_{v}$ adopted in CMC, and the intrinsic mixture fraction $f$ between each channel. Under specific assumptions about other uncertain aspects of isolated binary and globular cluster evolution, we report the median and $90 \%$ confidence interval of three physical parameters for the intrinsic population $\left(f, \alpha, r_{v}\right)=\left(0.20_{-0.18}^{+0.32}, 2.26_{-1.84}^{+2.65}, 2.71_{-1.17}^{+0.83}\right)$. This simultaneous constraint agrees with observed properties of globular clusters in the Milky Way and is an important first step in the pathway toward learning the astrophysics of compact binary formation through GW observations.
\end{abstract}

DOI: 10.1103/PhysRevD.103.083021

\section{INTRODUCTION}

The number of gravitational-wave (GW) detections is growing at an accelerating pace since the first detection [1]. The LIGO-Virgo-KAGRA Collaboration (LVKC) recently released the second Gravitational-Wave Transient Catalog (GWTC-2), which includes 39 events from the first half of the third observational run (O3) [2]. The number of GW events in this new catalogue (50) is $\sim 4$ times the number of events from the first two observational runs combined [3], allowing for the increasingly sensitive exploration of their mass, spin and merger redshift distributions [4]. As the statistical uncertainties in these distributions continue to drop with the growing number of detections, the population of $\mathrm{GW}$ events provides a unique, and increasingly powerful, avenue to probe a wide range of topics, including fundamental physics [5], cosmology [6,7], and astrophysics [8-10].

One of the most pressing questions in GW population analyses is which binary formation channels generate the

*kazewong@jhu.edu observed GW events. In particular, it is expected that compact binary mergers may arise via isolated binary evolution in the stellar field or dynamical assembly in dense clusters, although a variety of other channels have also been theorized [11-13]. In the isolated field scenario, compact binary mergers are the end result of stellar binary evolution $[14,15]$. On the other hand, events from dynamical formation scenarios are formed through multibody encounters in dense environments, such as globular clusters, young stellar clusters, or galactic nuclear clusters $[16,17]$. While the precise distributions of compact binaries originating from each scenario are not known, field and cluster channels generally differ in their predictions regarding spin, eccentricity, and component mass distributions of binary mergers. Due to mass transfer and tidal alignment, binaries resulting from the isolated formation tend to have component spins aligned with their orbital angular momenta, whereas dynamically formed binaries are believed to have an isotropic distribution of spins [18-21]. Also, hierarchical mergers are likely only possible in a dynamical-formation scenario [22-25], which could result in black holes with masses in the theorized pair-instability 
supernova (PISN) gap (commonly known as the "upper mass gap") [26,27].

Some studies have already sought to use the observed masses and spins of compact binary mergers to infer the mixing fraction between the two formation channels $[4,28,29]$. Such studies, however, generally adopt heuristic models for expected spin distributions, or ignore possible variation within individual channels that may be correlated with the inferred mixing fractions. Moreover, although the mixing fraction itself is an important question, understanding the mixing fraction alone yields little insight on the physics of each underlying formation channel. Thus, it is important to infer the mixing fraction and channelspecific parameters jointly. In this study, we create a mixture model of merging binary black holes (BBHs) from isolated binary evolution using the binary population synthesis code COSMIC and from globular clusters (GCs) using the $\mathrm{GC}$ evolution code CMC. We infer the properties of this mixture model by applying a deep learning enhanced hierarchical Bayesian modeling framework on the GWTC-2 BBH catalogue.

The paper is structured as follows: In Sec. II, we describe the library of COSMIC and CMC simulations used in this study. In Sec. III, we review our data analysis pipeline that applies deep-learning to emulate and interpolate between our library, allowing for hierarchical inference of parameters governing binary formation. In Sec. IV, we present results obtained by applying our pipeline to public data released in support of GWTC-2. Finally, in Sec. V, we discuss implications of this work.

\section{SIMULATION}

\section{A. Isolated binary evolution with COSMIC}

We generate a cosmological population of $\mathrm{BBH}$ mergers originating from isolated binary evolution using COSMIC [30], which is based on an updated version of BSE [31]. See [30] for a comprehensive summary of all upgrades currently employed in COSMIC and [32] for a detailed discussion of the prescriptions which most heavily impact compact-object formation in biaries. We assume that massive stars are initially distributed with masses following a power law with index $\alpha=-2.3$ [33] and 70\% of them have companions with mass ratios distributed uniformly [34]. (Corr: We distribute initial orbital periods with a power law in log orbital period with index $\pi=-0.55$ and $\log \left(P_{\text {orb }} /\right.$ day $) \in[0.15,5]$ following the assumptions of $[34,35]$ and initial eccentricities with a power law with index $\xi=-0.45$ for $e \in[0.001,0.9]$ [34]).

One of the largest uncertainties in binary evolution is the amount a binary's orbital separation shrinks as a result of common envelope evolution [36]. COSMIC employs the $\alpha \lambda$ prescription to parametrize how efficiently orbital energy is used in unbinding the stellar envelope, where $\lambda$ is the envelope binding energy and is calculated following
Appendix A of [37] assuming no contributions from ionizing energy. Previous studies suggest a wide range of ejection efficiencies varying from $\alpha=0.25-5$ for a wide variety of stellar masses (e.g., [38-40]). To capture this uncertainty, we ran 8 separate models $(\alpha=0.25,0.5,0.75$, $1,2,3,4,5)$ each with 16 metallicity bins spaced logarithmically between $Z_{\odot} / 200$ and $2 Z_{\odot}$, where $Z_{\odot}=$ 0.017 [41]. The binary evolution model, except for the variation of envelope ejection efficiency, is identical to that of [32] which consistently produces local $(z<0.01)$ merger rates consistent within a factor of 2 to the $90 \%$ credible interval of the observed rates from the GWTC-1 catalog for $\alpha=1,5$. For a discussion of the local comoving merger rates for our simulated populations, see Sec. V.

To generate a cosmological population of merging BBHs, we use the redshift-dependent star formation history and metallicity evolution of [42] and assume Planck 2015 cosmological parameters: $H_{0}=68 \mathrm{~km} \mathrm{~s}^{-1} \mathrm{Mpc}^{-1}, \Omega_{m}=$ 0.31 and $\Omega_{\Lambda}=0.69$ [43] as implemented in ASTROPY $[44,45]$. Similar to [32], we assume a truncated log-normal distribution of metallicities with $\sigma=0.5$ dex following [21]. We break the star formation into 100 linearly spaced redshift bins between 0 and 14 and calculate the number of BBHs formed, weighted by their metallicity, by normalizing the total mass of stars from our simulated population to the total amount of star formation in each redshift bin. We then record the lookback time and the redshift of each $\mathrm{BBH}$ merger to create a catalog of all merging BBHs for redshifts $z<15$.

\section{B. Globular cluster evolution with CMC}

We use $N$-body simulations presented in the CMC Cluster Catalog [46] to simulate GC evolution. These simulations were computed using CMC (for Cluster Monte Carlo) [47,48], a Hénon-type Monte Carlo code which includes various processes relevant to $\mathrm{BH}$ binary formation including two-body relaxation [47], three-body binary formation [49], direct integration of small- $N$ resonate encounters [50,51], and stellar/binary evolution. For the latter, CMC uses updated versions of SSE and BSE [31,52], identical to those used in COSMIC, only varying in choices of binary evolution prescriptions. [Corr: In CMC, we assume a 5\% percent primordial binary fraction (uniform across all primary masses which in turn are drawn from a Kroupa [53] initial mass function over the range $0.08-150 M_{\odot}$ ). We assume mass ratios are drawn from a uniform distribution in range [0.1-1]. Binary orbital periods are drawn from a distribution flat in logscale, with the orbital separations ranging from near contact ( $a \geq 5\left(R_{1}+R_{2}\right)$, where $R_{1}$ and $R_{2}$ are the stellar radii) to the hard/soft boundary of the cluster (reflecting the fact that soft binaries are expected to be broken quickly through dynamical encounters). Additionally, we assume initial eccentricities are drawn from a thermal distribution (e.g., [54]). 
In the CMC Cluster Catalog, four key cluster parameters are varied between the different simulations: initial number of stars per cluster $\left(N / 10^{5}=2,4,8,16\right)$, initial virial radius $\left(r_{v} / \mathrm{pc}=0.5,1,2,4\right)$, metallicity $\left(Z / Z_{\odot}=0.01\right.$, $0.1,1)$, and radial position within a (Milky-Way-like) galactic potential $\left(R_{\mathrm{gc}} / \mathrm{kpc}=2,8,20\right)$. Collectively, this simulation suite covers the full parameter space of the Milky Way GCs and captures the formation of a variety of astrophysical objects including GW sources, $\mathrm{x}$-ray binaries, millisecond pulsars, and blue stragglers.

By coupling a cluster age distribution model from [55] with the $\mathrm{BBH}$ merger delay time distributions gathered from the CMC models, a realistic distribution of dynamical BBH merger times can be assembled. In [46], this method was used to estimate a $\mathrm{BBH}$ merger rate of roughly $20 \mathrm{Gpc}^{-3} \mathrm{yr}^{-1}$ in the local universe (assuming all $N, r_{v}, Z$, and $R_{\mathrm{gc}}$ values are equally weighted), consistent with similar rate estimates from other recent studies (e.g., [56-58]).

While COSMIC can produce nearly arbitrarily large catalogs of compact binary mergers, the output of CMC is limited by the relatively high computational costs associated with dynamical $N$-body simulations (e.g., [48]). As a result, the total number of $\mathrm{GW}$ events from our set of CMC models is of order $10^{4}$ compared to several million from each COSMIC model. To mitigate the presence of small-scale fluctuations due to finite sampling, which may inadvertently be learned and reproduced by our emulator, we smooth the binary mass distributions given by each CMC simulation with a gaussian kernel using scipy.stats.gaussian_kde. The smoothing preserves physical features in the mass functions, such as highmass "bumps" due to repeated mergers, while smoothing out small unwanted scale fluctuations.

\section{Mixture model}

In an ideal scenario, a single model would be used to simultaneously predict both the observable properties and the merger rates of compact binaries across all different formation channels. In this case, the mixing fraction between channels would be a direct prediction of the model, requiring no additional free parameters. In practice, the compact binary merger rates given by both COSMIC and CMC are highly uncertain and possibly subject to severe systematic bias. Therefore, when combining simulations to create a mixture model between field and cluster populations, we deliberately introduce a free parameter $f$ controlling the mixing fraction:

$$
\begin{aligned}
p\left(m_{1}, m_{2}, z \mid \alpha, r_{v}, f\right)= & f p_{\text {cosmic }}\left(m_{1}, m_{2}, z \mid \alpha\right) \\
& +(1-f) p_{\mathrm{CMC}}\left(m_{1}, m_{2}, z \mid r_{v}\right),
\end{aligned}
$$

where $p_{\text {cosmic }}\left(m_{1}, m_{2}, z\right)$ and $p_{\mathrm{CMC}}\left(m_{1}, m_{2}, z\right)$ are the probability densities on the primary mass $m_{1}$, secondary
TABLE I. Event parameters and hyper-parameters used in this work.

Event parameters $\boldsymbol{\theta}$

$m_{1} \in[2.5,100] M_{\odot} \quad$ Source-frame primary mass of the binary $m_{2} \in[2.5,100] M_{\odot} \quad$ Source-frame secondary mass of the

$z \in[0,1.2] \quad \begin{gathered}\text { binary } \\ \text { Redshift of the binary }\end{gathered}$

Hyper-parameters $\lambda$

$\alpha \in[0.25,5] \quad$ Common envelope efficiency

$r_{v} \in[0.5,4] \mathrm{pc} \quad$ Initial cluster virial radius

$f \in[0,1] \quad$ Fraction of binaries from field-formation channel

mass $m_{2} \leq m_{1}$, and redshift $z$ of BBH mergers predicted by COSMIC and CMC, respectively. This choice of mixture model ensures that our conclusions are physically informed by the shapes of the observed mass and redshift distributions, and not on the rates of binary mergers. The parameters of the mixture model are summarized in Table I.

Each combination of hyper-parameters generically yields a different distribution of primary masses and mass ratios $\left(q=m_{2} / m_{1}\right)$. Several different examples are shown in Fig. 1, varying the common envelope efficiency $\alpha$ in COSMIC (left-hand side) and the initial cluster virial radius $r_{v}$ in CMC (right-hand side). Larger common envelope efficiencies, for instance, produce relatviely lower primary
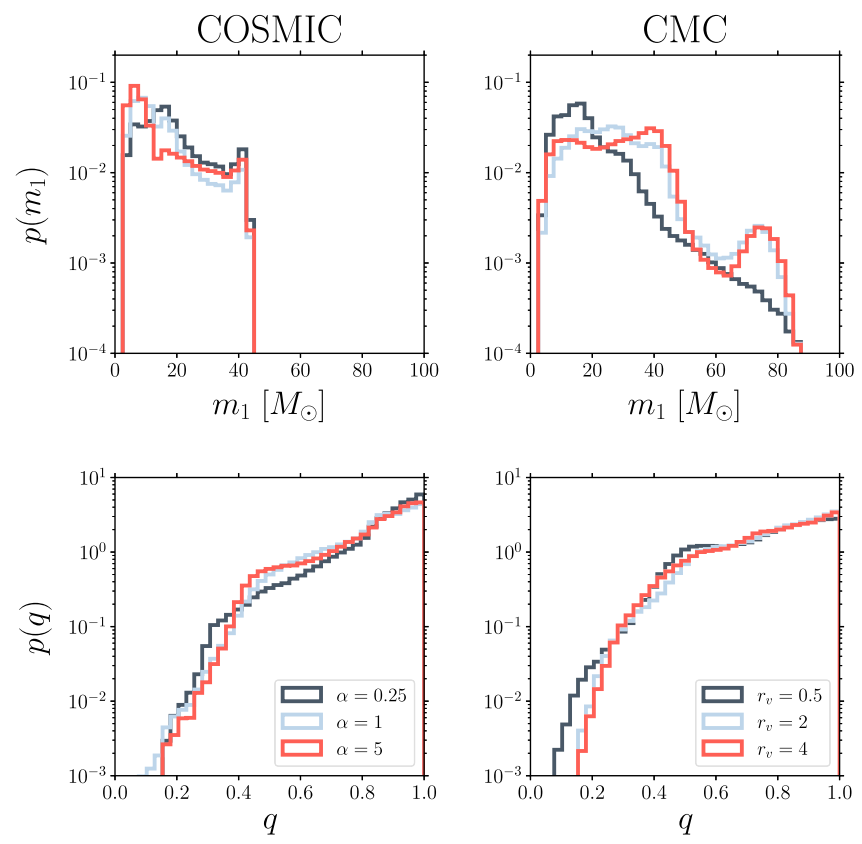

FIG. 1. Distributions of source-frame primary mass and mass ratio for all merging $\mathrm{BBHs}$ from the COSMIC and CMC models. The left column shows the distributions for different common envelope efficiencies and merging BBHs from COSMIC only, and the right column shows the distributions for different initial virial radii when we only consider $\mathrm{BBH}$ mergers from $\mathrm{CMC}$. 
masses, while clusters with larger $r_{v}$ retain more massive $\mathrm{BHs}$ at late times and thus exhibit the high-mass peak around $80 M_{\odot}$ due to repeated $\mathrm{BH}$ mergers.

The shift toward lower primary masses with increasing common envelope ejection efficiency is a result of increased rates of mergers during the common envelope phase, before a BBH forms. COSMIC assumes that $\mathrm{BH}$ mass is directly correlated with progenitor mass, thus lower mass BHs will have lower mass stellar progenitors which enter common envelopes in closer orbits relative to higher mass $\mathrm{BHs}$ and progenitors. Since the delay times for merging BBHs in all of our models which originate from postcommon-envelope binaries are short enough for the majority of the population to merge within a few Gyr at most (even with $\alpha=5$ ), the mergers of progenitors which would form lower mass BHs is the dominant effect which shapes the black hole primary mass spectrum. We note that Fig. 1 shows the probability distribution in primary mass, thus direct comparisons of histogram height do not reflect the absolute contributions of model to the overall rate of merging BBHs.

In the CMC models, the $\mathrm{BH}$ mass spectrum features three prominent peaks: the first at roughly $10-20 M_{\odot}$ due to the assumptions concerning mass fallback during core collapse (e.g., [59]), the second at roughly $40 M_{\odot}$ due to the assumptions concerning the pair-instability (e.g., [60]), and the third at roughly $70-80 M_{\odot}$ due to first-generation-BH-merger products retained in their host cluster postmerger (e.g., [24]). The first two peaks are similarly found in the COSMIC models (as expected, given these peaks are features of single star evolution assumptions), while the third peak is unique to the dynamical cluster environment. The shift toward higher primary masses at higher $r_{v}$ is a consequence of the effect of $r_{v}$ on $\mathrm{BH}$ cluster dynamics. Primarily due to mass segregation arguments, the most massive BHs in a cluster will, on average, be the first to be ejected from their host cluster and merge (e.g., [46,49]). The lower-mass $\left(\approx 10-15 M_{\odot}\right)$ BHs become dynamically active only after the most massive BHs have been ejected. For smaller initial $r_{v}$, high-mass $\mathrm{BHs}$ are dynamically processed and ejected early on. Therefore, in these clusters, high-mass $\left(M \gtrsim 40 M_{\odot}\right)$ BHs, including the second-generation BHs with masses in the pair-instability gap, typically merge at high redshift leaving only the least massive BHs in any significant quantity at late times (low redshift). Meanwhile, for high- $r_{v}$ clusters, the initial relaxation time is longer (e.g., [61]), so many high-mass $\mathrm{BHs}$ still remain and may merge at late times. Thus, $\mathrm{BBH}$ mergers tend to have higher component masses for higher $r_{v}$ clusters, as shown in Fig. 1.

The most significant difference between the two formation channels is the existence or absence of black holes in the upper mass gap, with $m_{1} \gtrsim 45 M_{\odot}$. All stars in our simulations, including those formed in GCs, are subject to PISNe which results in a sharp mass cutoff near
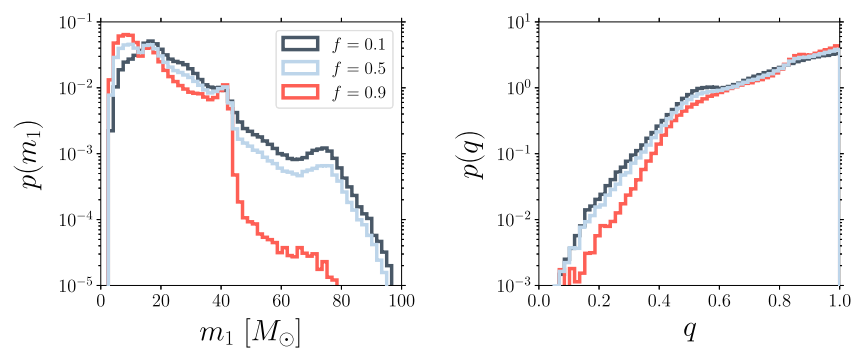

FIG. 2. The source-frame primary mass and mass ratio distribution for all merging BBHs in the mixture model. We fixed $\alpha=1$ and $r_{v}=1$ then mix the distributions with three values of the mixing fraction $f=[0.1,0.5,0.9]$

$m_{1} \approx 45 M_{\odot}$ for all first generation $\mathrm{BBH}$ mergers. We note that the assumptions for neutrino mass loss in our COSMIC and CMC models differ slightly such that the maximum mass for a first generation $\mathrm{BBH}$ component is $44.5 \mathrm{M}_{\odot}$ in COSMIC and $40.5 \mathrm{M}_{\odot}$ in CMC. This difference arises in the choice for neutrino mass loss to carry away a fixed $0.5 \mathrm{M}_{\odot}$ or $10 \%$ of the compact object mass at formation. Only hierarchical mergers, which do not occur in our isolated binary simulations, can result in BBHs with masses polluting the upper mass gap. This fact serves as the primary means of disentangling formation channels and measuring their mixing fraction $f$. Figure 2, for example, shows the total $m_{1}$ and $q$ distributions resulting from combining field and cluster channels, assuming alpha $=1$ and $r_{v}=1$ for several different mixing fractions $f$, the exact value of which sensitively controls the prevalence of high-mass systems. Thus the relative numbers of high- and low-mass binaries among GWTC-2 serves as a sensitive probe of the true value of $f$.

\section{METHOD}

In this section, we give a brief summary of the hierarchical inference and deep learning methods used in this work. We refer the readers to the comprehensive descriptions in e.g., Refs [62-64] for additional details.

Given data $\boldsymbol{d}$ spanning a number $N_{\text {obs }}$ of gravitationalwave detections, we wish to infer the posterior $p(\lambda \mid \boldsymbol{d})$ on the hyperparameters $\lambda$ governing their population. Assuming that the population is described as an inhomogenous Poisson process, the posterior takes the form $[64,65]$

$$
\begin{aligned}
p(\lambda \mid \boldsymbol{d}) \propto & \pi(\lambda) e^{-\alpha(\lambda) N(\lambda)} N(\lambda)^{N_{\mathrm{obs}}} \\
& \times \prod_{i=1}^{N_{\mathrm{obs}}} \int \frac{p\left(\boldsymbol{d} \mid \boldsymbol{\theta}_{\boldsymbol{i}}\right) p_{\mathrm{pop}}\left(\boldsymbol{\theta}_{\boldsymbol{i}} \mid \boldsymbol{\lambda}\right)}{\alpha\left(\boldsymbol{\theta}_{\boldsymbol{i}}\right)} \mathrm{d} \boldsymbol{\theta}_{\boldsymbol{i}},
\end{aligned}
$$

where $N(\lambda)$ is the intrinsic volume-integrated event rate predicted by the model, and $N_{\text {obs }}$ is the number of observed event in the data. $p\left(\boldsymbol{d} \mid \boldsymbol{\theta}_{\boldsymbol{i}}\right)$ is the likelihood of the $i$ th event in the observed catalogue and $\pi(\lambda)$ is the prior on our hyperparameters. Meanwhile, $p_{\text {pop }}\left(\boldsymbol{\theta}_{i} \mid \lambda\right)$ is the probability 
density function for the event-level parameters $\boldsymbol{\theta}_{\boldsymbol{i}}$; this function will be computed using a normalizing flow emulator.

The term $\alpha(\lambda)$ is known as the selection bias, and gives the fraction of events one expects to detect:

$$
\alpha(\lambda)=\int p_{\text {pop }}\left(\boldsymbol{\theta}^{\prime} \mid \lambda\right) P_{\operatorname{det}}\left(\boldsymbol{\theta}^{\prime}\right) d \boldsymbol{\theta}^{\prime}
$$

where $P_{\operatorname{det}}\left(\boldsymbol{\theta}^{\prime}\right)$ is the probability that an event with specific parameters $\boldsymbol{\theta}^{\prime}$ is successfully detected. In principle, one needs to inject a large amount of signals and recover them with a search pipeline to estimate the selection bias, which is very computationally expensive. In practice, we follow the procedure described in $[4,66]$, reweighting an injection campaign done by the LVKC to compute the selection bias for the $\mathrm{O} 12+\mathrm{O} 3 \mathrm{a}$ catalog. We interpolate $N(\lambda)$ and $\alpha(\lambda)$ and use the interpolated function during the inference to maximize computational efficiency.

As discussed above, the absolute merger rates predicted by the COSMIC and CMC simulations are likely subject to unknown systematic uncertainties. We therefore marginalize the posterior shown in Eq. (2) over the intrinsic merger rate, using a prior $\pi(N) \propto 1 / N$. Additionally, we do not know the underlying likelihood $p\left(\boldsymbol{d} \mid \boldsymbol{\theta}_{\boldsymbol{i}}\right)$ for each catalog event, but only the posterior $p\left(\boldsymbol{\theta}_{i} \mid \boldsymbol{d}\right)$ obtained under some default prior $\pi\left(\boldsymbol{\theta}_{i}\right)$ adopted by the LVKC during parameter estimation. Under both of these conditions, Eq. (2) can be written as [67]

$$
p(\lambda \mid \boldsymbol{d}) \propto \pi(\lambda) \prod_{i=1}^{N_{\mathrm{obs}}} \int \frac{p\left(\boldsymbol{\theta}_{\boldsymbol{i}} \mid \boldsymbol{d}\right)}{\pi\left(\boldsymbol{\theta}_{\boldsymbol{i}}\right)} \frac{p_{\mathrm{pop}}\left(\boldsymbol{\theta}_{\boldsymbol{i}} \mid \lambda\right)}{\alpha(\lambda)} \mathrm{d} \boldsymbol{\theta}_{\boldsymbol{i}}
$$

Specifically, the LVKC releases their event posterior in the form of discrete samples produced by their parameter estimation pipeline. Given these samples, the integral in Eq. (4) can be evaluated by using importance sampling, which turns the integral into a discrete sum over the event posterior PDF samples

$$
p(\lambda \mid \boldsymbol{d}) \propto \pi(\lambda) \prod_{i=1}^{N_{\mathrm{obs}}} \frac{1}{S_{i}} \sum_{j=1}^{S_{i}} \frac{p_{\mathrm{pop}}\left({ }^{j} \boldsymbol{\theta}_{i} \mid \lambda\right)}{\pi\left({ }^{j} \boldsymbol{\theta}_{i}\right) \alpha(\lambda)} .
$$

where $j$ labels the posterior samples of the $i$ th event and $S_{i}$ is the total number of samples per event. The default prior used in the LVKC LALINFERENCE software [68] is uniform in detector-frame masses and quadratic in luminosity distance, whereas we wish to model the distributions of source-frame masses and redshifts. In terms of these desired coordinates, the LALINFERENCE prior takes the form

$$
\pi\left(m_{1}, m_{2}, z\right) \propto(1+z)^{2} D_{L}^{2}(z)\left(D_{c}(z)+\frac{c(1+z)}{H(z)}\right) .
$$

To evaluate Eq. (5), we make use of the posterior samples presented in Refs. [3,2] and released through the Gravitational-Wave Open Science Center [69]. In particular, we use the "PublicationSamples" dataset associated with each event, and restrict to the 44 events with false alarm rates $<1 \mathrm{yr}^{-1}$ following Ref. [4].

The final term we need for evaluating Eq. (5) is the distribution of observables predicted by our simulation $p_{\text {pop }}(\boldsymbol{\theta} \mid \boldsymbol{\lambda})$, which we obtain through training a deep learning emulator on the simulations. Here we give the networkrelated hyperparameters for reproducing our result. A detailed discussion of the architecture of the neural network and of the training procedure can be found in Refs. [62,63]. We train a masked autoregressive flow network [70] with 10 hidden layers, each layer having 1024 units with ReLU activation. We include 3 observables $\left\{m_{1}, m_{2}, z\right\}$ and 3 hyper-parameters $\left\{f, \alpha, r_{v}\right\}$ in our training, as tabulated in Table I. The training set contains 160 simulations with different combinations of 8 values of $\alpha \in[0.25,5], 4$ values of $r_{v} \in[0.5,4]$ and 5 values of $f \in[0,1]$. Each simulation has $10^{5}$ events, of which $80 \%$ are randomly chosen our training set and $10 \%$ for both validation and test sets. Note that we follow the LVKC convention to enforce $m_{1}>m_{2}$. We train the network for 100 epoch on a Nvidia K80 GPU to ensure convergence. The code for the neural network is written in python with PYTORCH [71]. Equation (5) is then sampled using the MCMC package EMCEE [72] to produce the results shown in this work.

\section{RESULT}

We infer the hyper-parameters of Eq. (1) using the posterior samples publicly released in support of GWTC-1 [3,73] and GWTC-2 [2,74], restricting to the 44 events with false alarm rates $<1 \mathrm{yr}^{-1}$. Our main results are shown in Fig. 3. As shown in Fig. 1, a component mass larger than $\sim 45 M_{\odot}$ is a signature of GC-formed BBHs [Corr: within our two-channel model]. In GWTC-1, only GW170729 has a source-frame primary mass estimate with a median larger than $45 M_{\odot}$. With only 10 events in GWTC-1, the mixing fraction is therefore rather unconstrained, and the data show a preference for a near-equal mixing of the two populations instead of one dominating the other (grey 1-D histograms). This is not the case for GWTC-2. There are 8 events with median source-frame primary masses $>45 M_{\odot}$. Together with the events from GWTC-1, 9 out of 44 events pass the PISN mass gap. This boost in number of high mass events significantly shifts the preferred value of the mixing fraction toward the cluster formation scenario. [Corr: For comparison, if we ignore the 9 events in the PISN mass gap, the inferred mixing fraction becomes $0.5_{-0.28}^{+0.4}$.] Despite this preference for cluster formation, the field formation scenario can still contribute significantly to the entire observed population. Our $90 \%$ credible upper bound on the mixing fraction is $f=0.522$ 


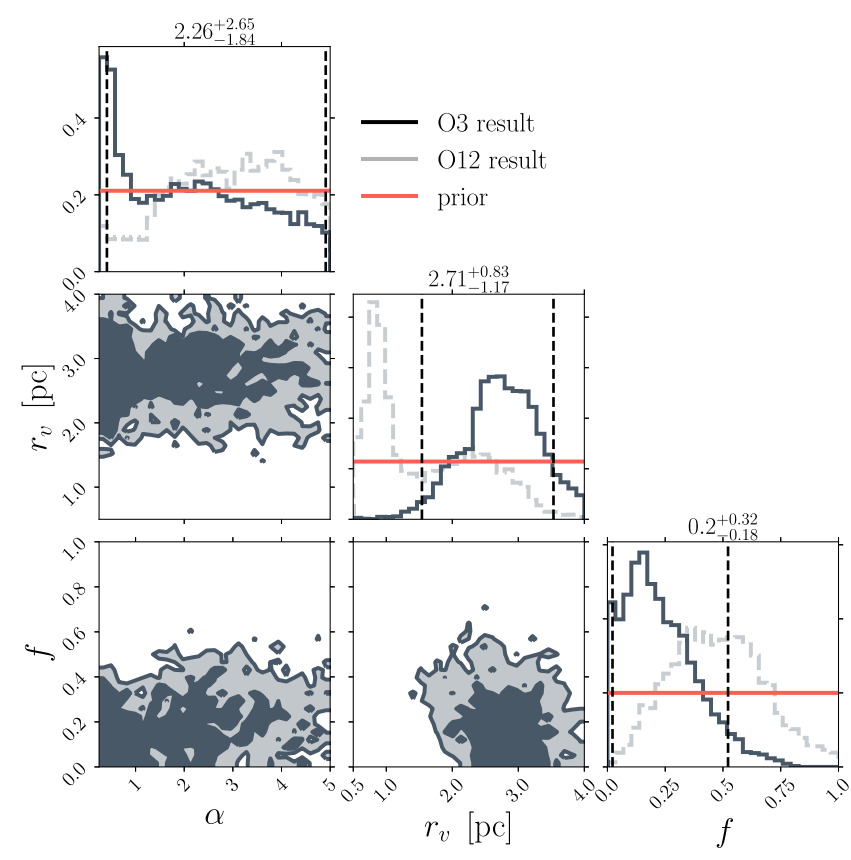

FIG. 3. The posterior distribution of our model inferred by using all BBHs up to GWTC-2. The contours represent $50 \%$ and $90 \%$ credible bounds. The grey lines in the 1D marginalized results are posterior distribution inferred by using only BBHs in GWTC-1.

suggesting that the contribution from the isolated channel could still surpass the cluster formation channel.

Our posterior on the common envelope efficiency $\alpha$ shows a mild preference toward lower values. Our relative insensitivity to $\alpha$ is due to two effects. First, varying $\alpha$ changes the mass distributions predicted by COSMIC by only a factor of a few, [Corr: so one need more events for the difference in distribution with difference $\alpha$ to become statistically significant.] Second, since the mixing fraction favors the cluster formation channel over the isolated formation channel, the effective number of events which was used to constrained the common envelope efficiency is even smaller.

In contrast, the initial cluster virial radius $r_{v}$ is reasonably well-constrained, since our module suggests that more $\mathrm{BBH}$ mergers originate in GCs than in isolation. There are 5 events with a component mass larger than $60 M_{\odot}$, which make up more than $10 \%$ of the entire dataset. As clusters with larger initial virial radii tend to yield more massive mergers at higher redshift (including hierarchical mergers with masses in the pair-instability gap), the excess of highmass events hints that the majority of clusters may have been born with moderately large virial radius (we predict a median value of $2.71 \mathrm{pc}$ ). [Corr: We also find the constraint on $r_{v}$ correlates with $f$ mildly. By taking a thin bin of posterior samples with $f=0$, we found the median and standard deviation of $r_{v}$ are $(2.79,0.44)$; With $f=0.5$, the median and standard deviation of $r_{v}$ are $(2.65,0.64)$. While the shift of median is rather insignificant, the posterior

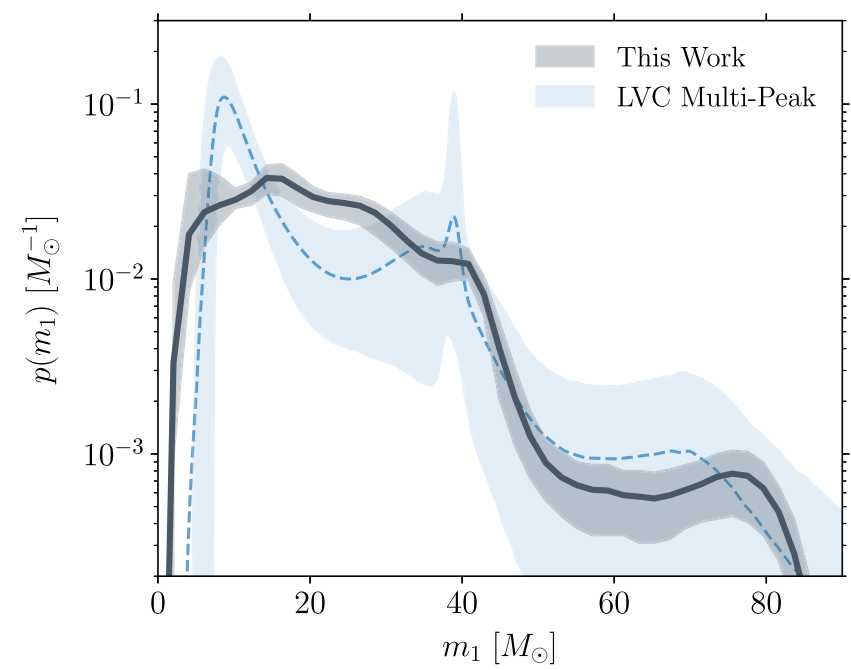

FIG. 4. The primary mass distribution (shaded grey) inferred by our mixture model. Specifically, the shaded band shows the central $90 \%$ credible bound on $p\left(m_{1}\right)$ as a function of primary mass, while the solid line marks the population predictive distribution: the inferred probability distribution on $m_{1}$ after marginalization over the field-cluster mixing fraction $f$, common envelope efficiency $\alpha$, and initial cluster virial radius $r_{v}$. For comparison, the shaded blue band marks the analogous result obtained by the LVC under their "multipeak" mass model; the dashed blue line is the corresponding population predictive distribution.

distribution is less constrained when mixed with COSMIC $(f>0)$ because we have less effective events to constrain CMC.]

Figure 4 shows our inferred primary mass distribution, marginalized over our posterior on $f, \alpha$, and $r_{v}$. For comparison, the light blue band indicates the mass spectrum inferred by the LVC using their phenomenological "multipeak" model, where the primary mass distribution is modeled as the superposition of a power law with two additional Gaussians that can capture the onset of pair instability and the presence of high-mass hierarchical mergers, if present [4]. Despite their very different parametrizations, both models yield qualitatively similar structure at moderate and high masses: a steep drop near $\sim 45 M_{\odot}$ (corresponding to the onset of the pair instability mass gap in our model) followed by a plateau and possible secondary peak near $80 M_{\odot}$ due to second-generation mergers in GCs.

\section{DISCUSSION}

Previous analyses (e.g., [46,75]) have shown a cluster's initial $r_{v}$ plays a prominent role in the cluster's longterm dynamical evolution. In particular, GCs born with smaller initial $r_{v}$ (and thus shorter relaxation times), are more likely to have undergone core collapse by the present day. In this case, the relative ratio of core-collapsed to noncore-collapsed GCs observed at present may provide a 
TABLE II. Event rates for each hyper-parameter and formation scenario. Rates are given for $z<0.1$ and in comoving volume per time.

\begin{tabular}{lcccccccc}
\hline \hline Isolated binaries & & & & & & & & \\
\hline$\alpha$ & 0.25 & 0.5 & 0.75 & 1 & 2 & 3 & 4 & 5 \\
$\Gamma_{\alpha}\left[\mathrm{Gpc}^{-3} \mathrm{yr}^{-1}\right]$ & 63.8 & 62.5 & 66.5 & 72.2 & 76.6 & 75.1 & 73.9 & 72.1 \\
Globular clusters & & & & & & & & \\
\hline$r_{v}[\mathrm{pc}]$ & 0.5 & & 1 & 2 & 4 & \\
$\Gamma_{r_{v}}\left[\mathrm{Gpc}^{-3} \mathrm{yr}^{-1}\right]$ & 31.6 & & 26.8 & & 20.9 & \multicolumn{2}{c}{8.7} & \\
\hline \hline
\end{tabular}

complementary constraint upon the initial $r_{v}$ distribution [46]. Taking the Milky Way GCs as a representative sample, roughly $80 \%$ of clusters have well-resolved cores (i.e., are non-core-collapsed) at present [76], potentially hinting that relatively large initial $r_{v}$ are typical, consistent with the predictions from our GWTC-2 inference.

There are a number of assumptions made in this work that can be improved upon in the future. To safeguard against systematic model uncertainties, we do not include merger rates in our inference. For completeness, we report the comoving merger rate at $z<0.1$ under each formation channel and hyper-parameter choice in Table II. The local merger rates from the isolated formation channel are lie near the maximum value of the $90 \%$ confidence intervals of the reported merger rates inferred from GWTC-2 of 23.9 $9_{-8.6}^{+14.9} \mathrm{Gpc}^{-3} \mathrm{yr}^{-1}$. This illustrates a potential systematic bias in the other parameters which define the isolated binary formation models. In particular, COSMIC assumes that natal kicks for BHs are drawn from a Maxwellian distribution with $\sigma=265 \mathrm{~km} / \mathrm{s}$ and then weighted by the amount of mass that falls back onto the proto-compactobject during formation [59]. If stronger kicks are assumed, BBHs with lower masses will preferentially be unbound, altering both the merger rate and mass distributions in the isolated formation scenario (e.g., [62,77,78]). COSMIC also assumes that stable Roche-overflow mass transfer is conservative, which may over predict the orbital evolution of a mass transferring binary (e.g., [79]). This is further compounded by our uncertain choice, following [80], of the mass ratios for which mass transfer is assumed to be dynamically unstable leading to a common envelope. If mass transfer proceeds stably for a wider range of mass ratios, fewer systems will experience dramatic orbital tightening during common envelope, potentially lowering predicted merger rates. We leave a full study of the combined effects of $\mathrm{BH}$ natal kicks, common envelope, mass transfer stability, and accretion efficiency to a future study.

Although the rate estimates from clusters shown in Table II match well the reported comoving rate inferred from GWTC-2, the cluster estimates also feature several uncertainties. A major uncertainty is the assumed cluster formation history. Observations of young stellar clusters in the local universe (e.g., [81]) indicate that initial cluster virial radii are roughly independent of cluster birth time and metallicity, however this is highly uncertain. If $r_{v}$ does vary with cluster birth time, it may substantially affect the conclusions of this study. Furthermore, the cluster birth time distribution itself is highly uncertain. Current theories of cluster formation fall into two main categories: clusters formed through active star formation (e.g., [55,82]) and clusters formed due to the collapse of dark matter halos during or before the epoch of reionization (e.g., [83]). Here, we have assumed the former scenario but if a large population of present-day GCs were instead born during reionization, our results may again change significantly. Future work should consider more carefully the various possible assumptions regarding cluster formation scenarios and the dependence of cluster properties such as $r_{v}$ on these various scenarios.

There are many valuable routes by which this present analysis may be expanded in the future. As features in the $\mathrm{BBH}$ spin distribution begin to be robustly resolved [4,84-88], future analyses can incorporate spin measurements alongside mass and redshift. Future measurements of orbital eccentricity may additionally help to discriminate between formation scenarios $[51,89,90]$. Finally, due to the still moderate number of events, in this work we restricted purely to BBHs and considered only CE and GC formation, neglecting other proposed formation channels such as primordial BHs, nuclear star clusters, open clusters, stellar triples, and AGN disks; future analysis can incorporate a greater diversity of formation scenarios, as in Ref. [88], and seek to self-consistently include neutron star and neutron star-black hole binaries alongside BBHs. We note that our results, which favor BBH formation in GCs, are in slight tension with those of Ref. [88], which identifies a preference for $\mathrm{CE}$ evolution. This tension may be due to a number of differences in the underlying models and data analysis methods used, including the methods for estimating selection effects (we adopt a pipeline-based false alarm threshold, compared to the optimal signal-to-noise threshold employed in Ref. [91]), the number of formation channels considered, the broader coverage of $r_{v}$ in our CMC models (our inferred value of $r_{v} \approx 2.7 \mathrm{pc}$ is outside the $r_{v}$ range considered in Ref. [91]), and our unique ability to continuously sample over otherwise discretized parameters like $\alpha$ and $r_{v}$.

To conclude, we have extracted the mixing fraction of BBHs formed in isolated binaries and GCs while simultaneously placing continuous constraints on the channelspecific hyper-parameters, notably the initial virial radius of GCs where the BBHs were formed. Our work marks an important milestone of learning astrophysics from populations of observed GW events. Instead of constraining a physics-agnostic phenomenological model, we use a population of GW events to place constraints on physical parameters such as mixing fraction directly. Furthermore, 
our model suggests that the GC properties inferred from the detected GW population are consistent with electromagnetic observations of present-day GCs, notably cluster core radii distributions. This result may be readily compared with other independent measurements to test theories of GC formation and evolution. As the number of GW detections increases in the future and theoretical models improve, one can apply the same methodology to discover and test a plethora of astrophysical theories more precisely.

\section{ACKNOWLEDGMENTS}

The authors are grateful to Simone Bavera, Vishal Baibhav, Christopher Berry, Emanuele Berti, Will Farr, Maya Fishbach, Vicky Kalogera, Ken K. Y. Ng, Isobel Romero-Shaw, Mike Zevin, and the referee for constructive feedback. K. W. K. W. is supported by NSF Grants No. PHY-1912550 and No. AST-2006538, NASA ATP
Grants No. 17-ATP17-0225 and No. 19-ATP19-0051, and NSF-XSEDE Grant No. PHY-090003. This research has made use of data, software and/or web tools obtained from the Gravitational Wave Open Science Center [92], a service of LIGO Laboratory, the LIGO Scientific Collaboration and the Virgo Collaboration. This research project was conducted using computational resources at the Maryland Advanced Research Computing Center (MARCC). The authors would like to acknowledge networking support by the GWverse COST Action CA16104, "Black holes, gravitational waves and fundamental physics. K. K. is supported by an NSF Astronomy and Astrophysics Postdoctoral Fellowship under Grant No. AST-2001751. The Flatiron Institute is supported by the Simons Foundation. Portions of this work were performed during the CCA LISA Sprint, supported by the Simons Foundation.
[1] B. P. Abbott et al. (LIGO Scientific and Virgo Collaborations), Phys. Rev. Lett. 116, 061102 (2016).

[2] R. Abbott et al. (LIGO Scientific and Virgo Collaborations), arXiv:2010.14527 [Phys. Rev. X (to be published)].

[3] B. P. Abbott et al. (LIGO Scientific and Virgo Collaborations), Phys. Rev. X 9, 031040 (2019).

[4] R. Abbott et al. (LIGO Scientific and Virgo Collaborations), arXiv:2010.14533.

[5] R. Abbott et al. (LIGO Scientific and Virgo Collaborations), arXiv:2010.14529.

[6] B. P. Abbott et al. (LIGO Scientific and Virgo Collaborations), Astrophys. J. 909, 218 (2021).

[7] A. Palmese et al. (DES Collaboration), Astrophys. J. 900, L33 (2020).

[8] R. Farmer, M. Renzo, S. de Mink, M. Fishbach, and S. Justham, Astrophys. J. 902, L36 (2020).

[9] Y. Bouffanais, M. Mapelli, F. Santoliquido, N. Giacobbo, G. Iorio, and G. Costa, arXiv:2010.11220.

[10] T. Callister, M. Fishbach, D. Holz, and W. Farr, Astrophys. J. Lett. 896, L32 (2020).

[11] S. Bird, I. Cholis, J. B. Muñoz, Y. Ali-Haïmoud, M. Kamionkowski, E. D. Kovetz, A. Raccanelli, and A. G. Riess, Phys. Rev. Lett. 116, 201301 (2016).

[12] F. Antonini, C. L. Rodriguez, C. Petrovich, and C. L. Fischer, Mon. Not. R. Astron. Soc. 480, L58 (2018).

[13] B. McKernan, K. E. Saavik Ford, J. Bellovary, N. W. C. Leigh, Z. Haiman, B. Kocsis, W. Lyra, M.-M. Mac Low, B. Metzger, M. O'Dowd, S. Endlich, and D. J. Rosen, Astrophys. J. 866, 66 (2018).

[14] M. Dominik, K. Belczynski, C. Fryer, D. Holz, E. Berti, T. Bulik, I. Mandel, and R. O'Shaughnessy, Astrophys. J. 759, 52 (2012).

[15] K. Belczynski et al., Astron. Astrophys. 636, A104 (2020).

[16] C. L. Rodriguez, P. Amaro-Seoane, S. Chatterjee, and F. A. Rasio, Phys. Rev. Lett. 120, 151101 (2018).
[17] U. N. Di Carlo, N. Giacobbo, M. Mapelli, M. Pasquato, M. Spera, L. Wang, and F. Haardt, Mon. Not. R. Astron. Soc. 487, 2947 (2019).

[18] C. L. Rodriguez, M. Zevin, C. Pankow, V. Kalogera, and F. A. Rasio, Astrophys. J. Lett. 832, L2 (2016).

[19] W. M. Farr, S. Stevenson, M. Coleman Miller, I. Mandel, B. Farr, and A. Vecchio, Nature (London) 548, 426 (2017).

[20] Y. Qin, T. Fragos, G. Meynet, J. Andrews, M. Sørensen, and H. Song, Astron. Astrophys. 616, A28 (2018).

[21] S. S. Bavera, T. Fragos, Y. Qin, E. Zapartas, C. J. Neijssel, I. Mandel, A. Batta, S. M. Gaebel, C. Kimball, and S. Stevenson, Astron. Astrophys. 635, A97 (2020).

[22] D. Gerosa and E. Berti, Phys. Rev. D 95, 124046 (2017).

[23] Z. Doctor, D. Wysocki, R. O'Shaughnessy, D. E. Holz, and B. Farr, arXiv:1911.04424.

[24] C. L. Rodriguez, M. Zevin, P. Amaro-Seoane, S. Chatterjee, K. Kremer, F. A. Rasio, and C. S. Ye, Phys. Rev. D 100, 043027 (2019).

[25] B. McKernan, K. E. S. Ford, R. O'Shaugnessy, and D. Wysocki, Mon. Not. R. Astron. Soc. 494, 1203 (2020).

[26] S. Woosley, Astrophys. J. 836, 244 (2017).

[27] R. Farmer, M. Renzo, S. de Mink, P. Marchant, and S. Justham, arXiv:1910.12874.

[28] Y. Bouffanais, M. Mapelli, D. Gerosa, U. N. Di Carlo, N. Giacobbo, E. Berti, and V. Baibhav, Astrophys. J. 886 (2019).

[29] M. Safarzadeh, Astrophys. J. Lett. 892, L8 (2020).

[30] K. Breivik, S. Coughlin, M. Zevin, C. L. Rodriguez, K. Kremer, C. S. Ye, J. J. Andrews, M. Kurkowski, M. C. Digman, S. L. Larson, and F. A. Rasio, Astrophys. J. 898, 71 (2020).

[31] J. R. Hurley, C. A. Tout, and O. R. Pols, Mon. Not. R. Astron. Soc. 329, 897 (2002).

[32] M. Zevin, M. Spera, C. P. L. Berry, and V. Kalogera, Astrophys. J. Lett. 899, L1 (2020). 
[33] P. Kroupa, Mon. Not. R. Astron. Soc. 322, 231 (2001).

[34] H. Sana, S. E. de Mink, A. de Koter, N. Langer, C. J. Evans, M. Gieles, E. Gosset, R. G. Izzard, J. B. Le Bouquin, and F. R. N. Schneider, Science 337, 444 (2012).

[35] M. Renzo, E. Zapartas, S. E. de Mink, Y. Götberg, S. Justham, R. J. Farmer, R. G. Izzard, S. Toonen, and H. Sana, Astron. Astrophys. 624, A66 (2019).

[36] N. Ivanova, S. Justham, X. Chen, O. De Marco, C. L. Fryer, E. Gaburov, H. Ge, E. Glebbeek, Z. Han, X. D. Li, G. Lu, T. Marsh, P. Podsiadlowski, A. Potter, N. Soker, R. Taam, T. M. Tauris, E. P. J. van den Heuvel, and R. F. Webbink, Astron. Astrophys. Rev. 21, 59 (2013).

[37] J. S. W. Claeys, O. R. Pols, R. G. Izzard, J. Vink, and F. W. M. Verbunt, Astron. Astrophys. 563, A83 (2014).

[38] M. Zorotovic, M. R. Schreiber, B. T. Gänsicke, and A. Nebot Gómez-Morán, Astron. Astrophys. 520, A86 (2010).

[39] O. De Marco, J.-C. Passy, M. Moe, F. Herwig, M.-M. Mac Low, and B. Paxton, Mon. Not. R. Astron. Soc. 411, 2277 (2011).

[40] T. Fragos, J. J. Andrews, E. Ramirez-Ruiz, G. Meynet, V. Kalogera, R. E. Taam, and A. Zezas, Astrophys. J. Lett. 883, L45 (2019).

[41] N. Grevesse and A. J. Sauval, Space Sci. Rev. 85, 161 (1998).

[42] P. Madau and T. Fragos, Astrophys. J. 840, 39 (2017).

[43] P. A. Ade et al. (Planck Collaboration), Astron. Astrophys. 594, A13 (2016).

[44] T. P. Robitaille, E. J. Tollerud et al. (Astropy Collaboration), Astron. Astrophys. 558, A33 (2013).

[45] A. M. Price-Whelan, B. M. SipHocz et al. (Astropy Collaboration), Astron. J. 156, 18 (2018).

[46] K. Kremer, C. S. Ye, N. Z. Rui, N. C. Weatherford, S. Chatterjee, G. Fragione, C. L. Rodriguez, M. Spera, and F. A. Rasio, Astrophys. J. Suppl. Ser. 247, 48 (2020).

[47] K. J. Joshi, F. A. Rasio, S. P. Zwart, and S. Portegies Zwart, Astrophys. J. 540, 969 (2000).

[48] B. Pattabiraman, S. Umbreit, W.-k. Liao, A. Choudhary, V. Kalogera, G. Memik, and F. A. Rasio, Astrophys. J. Suppl. Ser. 204, 15 (2013).

[49] M. Morscher, B. Pattabiraman, C. Rodriguez, F. A. Rasio, and S. Umbreit, Astrophys. J. 800, 9 (2015).

[50] J. M. Fregeau and F. A. Rasio, Astrophys. J. 658, 1047 (2007).

[51] C. L. Rodriguez, P. Amaro-Seoane, S. Chatterjee, and F. A. Rasio, Phys. Rev. Lett. 120, 151101 (2018).

[52] J. R. Hurley, O. R. Pols, and C. A. Tout, Mon. Not. R. Astron. Soc. 315, 543 (2000).

[53] P. Kroupa, Mon. Not. R. Astron. Soc. 322, 231 (2001).

[54] D. C. Heggie, Mon. Not. R. Astron. Soc. 173, 729 (1975).

[55] K. El-Badry, E. Quataert, D. R. Weisz, N. Choksi, and M. Boylan-Kolchin, Mon. Not. R. Astron. Soc. 482, 4528 (2019).

[56] C. L. Rodriguez and A. Loeb, Astrophys. J. Lett. 866, L5 (2018).

[57] G. Fragione and B. Kocsis, Phys. Rev. Lett. 121, 161103 (2018).

[58] F. Antonini and M. Gieles, Phys. Rev. D 102, 123016 (2020).

[59] C. L. Fryer, K. Belczynski, G. Wiktorowicz, M. Dominik, V. Kalogera, and D. E. Holz, Astrophys. J. 749, 91 (2012).
[60] K. Belczynski, A. Heger, W. Gladysz, A. J. Ruiter, S. Woosley, G. Wiktorowicz, H. Y. Chen, T. Bulik, R. O'Shaughnessy, D. E. Holz, C. L. Fryer, and E. Berti, Astron. Astrophys. 594, A97 (2016).

[61] J. Binney and S. Tremaine, Galactic Dynamics (Princeton University Press, Princeton, 2008), https://ui.adsabs.harvard .edu/abs/2008gady.book.....B/abstract.

[62] K. W. K. Wong and D. Gerosa, Phys. Rev. D 100, 083015 (2019).

[63] K. W. Wong, G. Contardo, and S. Ho, Phys. Rev. D 101, 123005 (2020).

[64] I. Mandel, W. M. Farr, and J. R. Gair, Mon. Not. R. Astron. Soc. 486, 1086 (2019).

[65] T. J. Loredo, AIP Conf. Proc. 735, 195 (2004).

[66] W. M. Farr, Res. Notes Am. Astron. Soc. 3, 66 (2019).

[67] M. Fishbach, D. E. Holz, and W. M. Farr, Astrophys. J. Lett. 863, L41 (2018).

[68] J. Veitch, V. Raymond, B. Farr, W. Farr, P. Graff, S. Vitale, B. Aylott, K. Blackburn, N. Christensen, M. Coughlin, W. Del Pozzo, F. Feroz, J. Gair, C. J. Haster, V. Kalogera, T. Littenberg, I. Mandel, R. O'Shaughnessy, M. Pitkin, C. Rodriguez, C. Röver, T. Sidery, R. Smith, M. Van Der Sluys, A. Vecchio, W. Vousden, and L. Wade, Phys. Rev. D 91, 042003 (2015).

[69] M. Vallisneri, J. Kanner, R. Williams, A. Weinstein, and B. Stephens, in Journal of Physics Conference Series, Vol. 610 (2015), p. 012021 [arXiv:1410.4839].

[70] G. Papamakarios, T. Pavlakou, and I. Murray, arXiv:1705 .07057 .

[71] A. Paszke, S. Gross, S. Chintala, G. Chanan, E. Yang, Z. DeVito, Z. Lin, A. Desmaison, L. Antiga, and A. Lerer, arXiv:1912.01703.

[72] D. Foreman-Mackey, D. W. Hogg, D. Lang, and J. Goodman, Publ. Astron. Soc. Pac. 125, 306 (2013).

[73] LIGO Scientific and Virgo Collaborations, https://doi.org/ 10.7935/KSX7-QQ51.

[74] LIGO Scientific and Virgo Collaborations, https://doi.org/ LIGO-P2000223/public.

[75] K. Kremer, S. Chatterjee, C. S. Ye, C. L. Rodriguez, and F. A. Rasio, Astrophys. J. 871, 38 (2019).

[76] W. E. Harris, Astron. J. 112, 1487 (1996).

[77] K. Belczynski, V. Kalogera, and T. Bulik, Astrophys. J. 572, 407 (2002).

[78] K. Belczynski et al., Astron. Astrophys. 636, A104 (2020).

[79] L. A. C. van Son, S. E. De Mink, F. S. Broekgaarden, M. Renzo, S. Justham, E. Laplace, J. Morán-Fraile, D. D. Hendriks, and R. Farmer, Astrophys. J. 897, 100 (2020).

[80] K. Belczynski, V. Kalogera, F. A. Rasio, R. E. Taam, A. Zezas, T. Bulik, T. J. Maccarone, and N. Ivanova, Astrophys. J. Suppl. Ser. 174, 223 (2008).

[81] S. F. Portegies Zwart, S. L. W. McMillan, and M. Gieles, Annu. Rev. Astron. Astrophys. 48, 431 (2010).

[82] J. M. D. Kruijssen, Mon. Not. R. Astron. Soc. 454, 1658 (2015).

[83] S. M. Fall and M. J. Rees, Astrophys. J. 298, 18 (1985).

[84] S. Vitale, R. Lynch, R. Sturani, and P. Graff, Classical Quantum Gravity 34, 03LT01 (2017).

[85] S. Stevenson, C. P. L. Berry, and I. Mandel, Mon. Not. R. Astron. Soc. 471, 2801 (2017).

[86] C. Talbot and E. Thrane, Phys. Rev. D 96, 023012 (2017). 
[87] S. S. Bavera, T. Fragos, M. Zevin, C. P. L. Berry, P. Marchant, J. J. Andrews, S. Coughlin, A. Dotter, K. Kovlakas, D. Misra, J. G. Serra-Perez, Y. Qin, K. A. Rocha, J. Román-Garza, N. H. Tran, and E. Zapartas, arXiv:2010.16333.

[88] M. Zevin, S. S. Bavera, C. P. Berry, V. Kalogera, T. Fragos, P. Marchant, C. L. Rodriguez, F. Antonini, D. E. Holz, and C. Pankow, arXiv:2011.10057.
[89] J. Samsing, Phys. Rev. D 97, 103014 (2018).

[90] I. M. Romero-Shaw, P. D. Lasky, and E. Thrane, Mon. Not. R. Astron. Soc. 490, 5210 (2019).

[91] M. Zevin, C. Pankow, C. L. Rodriguez, L. Sampson, E. Chase, V. Kalogera, and F. A. Rasio, Astrophys. J. 846, 82 (2017).

[92] https://www.gw-openscience.org 